\title{
Polyphyllin I inhibits the growth of ovarian cancer cells in nude mice
}

\author{
LINHUI GU ${ }^{1,2}$, JIANGUO FENG ${ }^{1,2}$, ZHIGUO ZHENG ${ }^{1,2}$, HAIYAN XU ${ }^{3}$ and WEI YU ${ }^{3}$ \\ ${ }^{1}$ Research Institute; ${ }^{2}$ Key Laboratory of Diagnosis and Treatment Technology on Thoracic Oncology; \\ ${ }^{3}$ Biological Specimen Bank, Zhejiang Cancer Hospital, Hangzhou, Zhejiang 310022, P.R. China
}

Received April 24, 2015; Accepted September 6, 2016

DOI: $10.3892 / \mathrm{ol} .2016 .5348$

\begin{abstract}
Polyphyllin I (PPI) is an active component in Rhizoma Paridis, which displays extensive pharmacological antitumor activities. In a previous study, we found that polyphyllin I exhibited inhibitory effects on cell growth in the human ovarian cancer HO-8910PM cell line, as well as promoting apoptosis and the inhibition of cell migration. Furthermore, gene expression was also profiled by microarray, which showed that numerous genes were altered by PPI; three genes were of particular note that were associated with tumor progression, namely, Caspase-9, C-jun and Wnt5a. In the present study, the effect of PPI on subcutaneous tumor growth (HO-8910PM cells) in nude mice was further evaluated, and immunohistochemistry and reverse transcription polymerase chain reaction (RT-PCR) were used to examine the expression of Caspase-9, C-jun and Wnt5a in subcutaneous and lung metastatic tumor tissues, in order to investigate the possible mechanisms involved. The results showed that PPI significantly inhibited the tumor growth in vivo without a marked impact on body weight, and through use of immunohistochemical staining and RT-PCR, it was found that the expression of Caspase-9 and Wnt5a was decreased, while the expression of $\mathrm{C}$-jun was increased, in subcutaneous and lung metastatic tumor tissue; this was consistent with the in vitro results. In conclusion, the present study showed that PPI exerted antitumor activity on ovarian cancer cells in vivo, and indicated that the modulation of Caspase-9, C-jun and Wnt5a may be involved in the antitumor effect of PPI.
\end{abstract}

\section{Introduction}

Ovarian cancer is one of the most frequently occurring malignant tumors in the female reproductive organs, with its

Correspondence to: Dr Linhui Gu, Research Institute, Zhejiang Cancer Hospital, 38 Guangji Road, Hangzhou, Zhejiang 310022, P.R. China

E-mail: linhuigu@yeah.net

Key words: polyphyllin I, ovarian cancer, growth, C-jun, Caspase-9, Wnt5a morbidity rate ranked third, just behind cervical cancer and endometrial cancer, but ranking first among the gynecological tumors. Since the onset of ovarian cancer is not clear and without specific symptoms at the early stage, the majority of clinical cases are detected at the advanced stage. Although patients may initially experience good post-operative reactions, they finally succumb to recurrence and metastasis, and the 5-year survival rate is only $25-30 \%(1,2)$.

Rhizoma Paridis is the rhizome of Paris polyphylla Smith var. chinensis (Franch) Hara, which belongs to the Liliaceae family. In traditional Chinese medicine, the root is suggested to exhibit effects that include heat-clearance, detoxification, the relief of swelling and pain, the cooling of the liver and the arrest of convulsions. Modern pharmacological studies have shown that Rhizoma Paridis displays extensive pharmacological antitumor, immune regulation and cardiovascular effects, and is generally used in treating malignant lymphoma, lung cancer, nasopharyngeal carcinoma, cerebral tumors and digestive system neoplasms. It has previously been found that Paris saponins and extracts of Rhizoma Paridis show significant antitumor activity in in vivo and in vitro experiments, with multiple targets and pathways. The mechanism may be associated with its own cytotoxicity and its effects in accelerating apoptosis, affecting the cell cycle of tumor cells, inhibiting the generation of the tumor vasculature and regulating immune function $(3,4)$.

In a previous in vitro study, we demonstrated that polyphyllin I (PPI) resulted in growth inhibition, accelerated apoptosis and anti-metastasis in a highly metastatic human ovarian cancer HO-8910PM cell line (5). Simultaneously, screening of a gene chip was conducted and it was found that compared with the control group, there were 123 differentially-expressed genes in the cells treated by PPI; 70 were downregulated and 53 were upregulated, and they were associated with processes such as apoptosis, proliferation, migration, invasion and angiogenesis. C-jun, Caspase- 9 and Wnt5A in particular were validated at the gene and protein level. The expression of Caspase-9 and Wnt5a decreased with an increased dosage of PPI, and the dose-effect association was marked. By contrast, the expression of $\mathrm{C}$-jun increased with increased dosage and lengthened active time of the drug (6).

The main purpose of the present study was to observe the in vivo antitumor effects of PPI and analyze the changed expression of key molecules, such as C-jun, Caspase-9 and 
Wnt5a, which were identified in previous in vitro studies. The study aimed to discuss whether these molecules are involved in the antitumor effect of PPI and the molecular mechanism involved by a further nude mouse tumorigenicity assay in vivo.

\section{Materials and methods}

Experimental reagents. PPI (National Institute for the Control of Pharmaceutical and Biological Products, Beijing, China; batch number, 111590-200402), cis-platinum (DDP; Yunnan, China), reverse transcription-polymerase chain reaction (RT-PCR) kit (PrimeScript ${ }^{\mathrm{TM}}$ RT reagent kit; Takara Biotechnology Co., Ltd., Dalian, China), primers (synthesized by Takara Biotechnology Co., Ltd.) and PCR kit (SYBR Premix Ex Taq ${ }^{\mathrm{TM}}$ II: catalog no., DRR820A; Dalian, China) were used in the present study, as well as primary antibodies against Caspase-9 (ab32539), C-jun (ab31419) and Wnt5a (ab72583), which were obtained from Abcam (Cambridge, UK) and used at 1:80 dilution. The secondary antibody, which was the working solution from the AuraStain SP Mouse/Rabbit IHC test kit (P003IH-1) was acquired from Auragene Bioscience Corporation, Inc. (Changsha, China).

In vivo animal experiments. A total of 32 female BALB/c nude mice (4 weeks old, weighing 17-20 g) were obtained from Shanghai Slack Laboratory Animal Co., Ltd. (Shanghai, China; license no., SCXK (Shanghai) 2007-0005). The animals were maintained in a specific pathogen-free environment. The animal experiments were approved by the Institutional Animal Care and Use Committee of Zhejiang Chinese Medicine University (Hangzhou, Zhejiang, China).

Nude mouse HO-8910PM transplantation tumor mass, which was generated in our previous study, was inoculated subcutaneously into the back of nude mouse (7). The mass was cut after it grew up and $0.5-1 \mathrm{~cm}^{3}$ of the mass was inoculated subcutaneously into the back of the right upper extremity of each nude mouse. It took 2 weeks for the mass to grow to soybean-size $\left(0.3 \mathrm{~cm}^{3}\right)$. The mice were then randomly assigned into 4 groups (8 mice in each group) as follows: Saline group (containing the same quantity of dimethyl sulfoxide, $1.5 \mu \mathrm{l} / \mathrm{ml})$, PPI low-concentration group (1.5 mg/ $\mathrm{kg}$ PPI), PPI high-concentration group ( $3 \mathrm{mg} / \mathrm{kg}$ PPI) and DDP-positive control group ( $2 \mathrm{mg} / \mathrm{kg})$. DDP is widely used in ovarian cancer; thus, it was used in the present study as a positive control. The volume of the drug for intraperitoneal injection was limited at $1 \%$ of the body weight, and the drug was administered every 7 days for 7 weeks in total. The maximum longitudinal diameter (a) and transverse diameter (b) of the transplant subcutaneous sarcoma were measured using Vernier calipers every 7 days. The mean tumor volume of each group was determined according to $V=1 / 2 a^{2}$ and the growth curves were drawn. The mice were also weighed on an electronic scale every 7 days.

Histological experiment. After 49 days, the animals were sacrificed by cervical dislocation and dissections were performed to obtain hearts, livers, spleens, lungs and kidneys. White metastases the size of rice grains could be observed by the naked eye in the lungs, but there were no abnormalities observed in other organs. The organs were divided into two
Table I. Primer sequences for target genes.

\begin{tabular}{lc}
\hline Gene name & Primer sequences \\
\hline$\beta$-actin & \\
Forward & 5'-TGGCACCCAGCACAATGAA-3' \\
Reverse & 5'-CTAAGTCATAGTCCGCCTAGAAGCA-3' \\
C-jun & \\
Forward & 5'-CACGTGAAGTGACGGACTGTTCTA-3' \\
Reverse & 5'-CAGGGTCATGCTCTGTTTCAGG-3' \\
Wnt5a & \\
Forward & 5'-TTCGCCCAGGTTGTAATTGAAG-3' \\
Reverse & 5'-CTGCATGTGGTCCTGATACAAGTG-3' \\
Caspase-9 & \\
Forward & 5'-GCCATATCTAGTTTGCCCACACC-3' \\
Reverse & 5'-CACTGCTCAAAGATGTCGTCCA-3'
\end{tabular}

sections, one of which was stored in liquid nitrogen, while the other was added to $10 \%$ neutral buffered formalin ( $\mathrm{pH}$ 7.4) prior to tissue chips $(2 \mathrm{~mm}$ ) being made by Google Organisms Technology Co., Ltd. (Wuhan, China). Hematoxylin and eosin staining was performed on the sections and an analysis of the tissue chips was performed using iViewer (Beijing Unic-tech Co., Ltd., Beijing, China).

Immunohistochemistry. The expression conditions of Caspase-9, C-jun and Wnt5a proteins of subcutaneous tumor tissues and lung metastatic tumor tissue with metastasis were analyzed. Paraffin-embedded tissue chips were treated according to immunohistochemical processes, and antigen and antibody were bound together through high-pressure thermal remediation. Rabbit anti-Caspase-9, C-jun and Wnt5a were all used at 1:80 dilution (Abcam). The subsequent procedures would be performed as per the instructions of the AuraStain SP Mouse/Rabbit IHC test kit (Auragene Bioscience, Inc.). Staining amount (A) was scored as follows: 0 , no stained cells; 1, $1-10 \%$ stained cells; 2, 11-50\% stained cells; 3, 51-80\% stained cells; 4, 81-100\% stained cells. Staining intensity score (B) was scored as follows: 0, negative; 1, weakly-positive; 2 , moderately-positive; 3 , strongly-positive. The immunohistochemistry staining score (HIS) was calculated as follows: HIS = A x B. HIS of 9-12, strongly-positive; 5-8, moderately-positive; 1-4, weakly-positive; and 0 , negative.

$R T-P C R$. The tissue was ground with liquid nitrogen and TRIzol was added to extract RNA by a conventional method (8). The RNA was reverse-transcribed into cDNA according to the instructions of the RT-PCR kit. Quantitative PCR was conducted on an Applied Biosystems ${ }^{\circledR} 7500$ Real-Time PCR System (Thermo Fisher Scientific, Inc., Waltham, MA, USA) using SYBR Green with specific primers (Table I). The PCR amplifications conditions were as follows: $95^{\circ} \mathrm{C}$ for $10 \mathrm{~min}$, $95^{\circ} \mathrm{C}$ for $15 \mathrm{sec}$ and $60^{\circ} \mathrm{C}$ for $1 \mathrm{~min}$ (for 40 cycles). Using a solubility curve to check whether there was non-specific banding in the PCR amplified products, the amplification conditions were as follows: $95^{\circ} \mathrm{C}$ for $15 \mathrm{sec}$ and $60^{\circ} \mathrm{C}$ for $1 \mathrm{~min}$, for 40 cycles. The $\mathrm{Cq}$ value was automatically generated and 

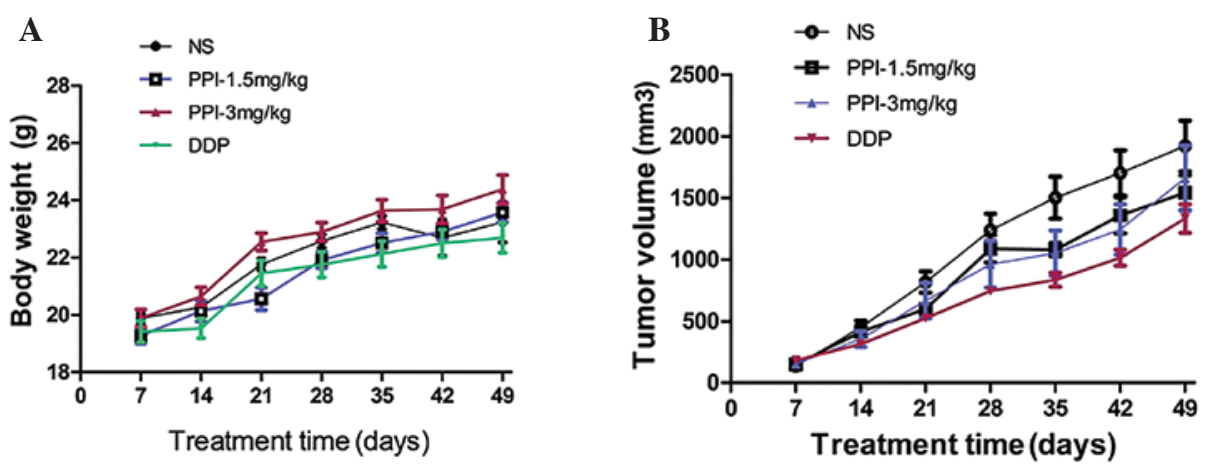

C

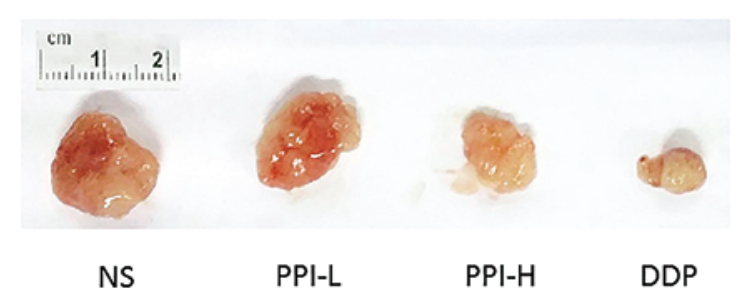

Figure 1. PPI inhibits the growth of ovarian cancer cells in nude mice. (A) Body weight was measured every 7 days, and expressed as the mean \pm standard deviation. There was a significant difference between the DDP and PPI-H groups $(\mathrm{P}=0.02)$. (B) Tumor volume was measured every 7 days. There was a significant difference between the NS and PPI-H/DDP groups, with $\mathrm{P}=0.0370$ and $\mathrm{P}=0.008$, respectively. (C) Representative images for each experimental group. PPI, polyphyllin I; NS, normal saline; DDP, cis-platinum; PPI-H, PPI high concentration; PPI-L, PPI low concentration.

analyzed. The relative expression of the genes was calculated using the $2^{-\Delta \Delta \mathrm{Cq}}$ method (9). The experiment was repeated three times.

Data processing and statistical analysis. The data were processed by SPSS18.0 software (IBM SPSS, Armonk, NY, USA). The relative expression was represented as the mean plus standard deviation. A one-way analysis of variance and least significant difference test was used to compare the normally distributed data between groups, with the non-parametric method used to test the abnormally distributed data. Differences were statistically significant between each group when $\mathrm{P}<0.05$.

\section{Results}

Effects of PPI on body weight and tumor growth. Within 7 weeks, the body weights of the nude mice in the groups differed. During the experiment, there was 1 mouse in the PPI high concentration group that died on day 40, while the others all survived. The toxic effect in the DDP group was the most significant, with reduced activity and declined food intake; in the other groups, the mice were as normal. The body weight of the mice in the DDP and NS groups increased at first and then decreased, particularly in the DDP group. In the PPI groups with different dosages, the body weight increased continuously, but without a significant difference compared with the control group. There was a significant difference between the DDP and PPI high concentration groups $(\mathrm{P}=0.02)$ (Fig. 1A).

The growth of transplant subcutaneous sarcoma in each group showed a rising trend by varying degrees following administration, being fastest in the NS group and slowest in the DDP group. There was a significant difference in the size of the tumor among the four groups $(\mathrm{F}=3.418, \mathrm{P}=0.038)$. When the NS group was compared with PPI high concentration and DDP groups, there were significant differences $(\mathrm{P}=0.0370$ and $\mathrm{P}=0.008$, respectively) (Fig. $1 \mathrm{~B}$ ). It could be observed that in the PPI high and low concentration groups, the tumor sizes were significant smaller than that in the NS control group, and in the DDP-positive group, the tumor size was the smallest (Fig. 1C).

Caspase-9, C-jun and Wnt5a protein expression. In our previous in vitro experiment, it was noted that three key molecules (Caspase-9, C-jun and Wnt5a) were regulated by PPI, which may be associated with the antitumor effect. Therefore, in vivo experiments were performed in the present study for confirmation. The immunohistochemical results showed that the expression of Caspase-9 was decreased in the PPI groups in the tumor tissue, and that Wnt5a expression was also decreased in the PPI groups, while C-jun expression was increased (Fig. 2).

Similarly, the change tendency was the same in the lung metastasis tumor tissue, in which the expression of Caspase-9 and Wnt5a was also decreased in PPI groups, while that of C-jun was increased (Fig. 3).

C-jun, Caspase-9 and Wnt5a gene expression. Furthermore, the changes in $\mathrm{C}$-jun, Caspase-9 and Wnt5a gene expression were also detected at the transcriptional level. The results showed that there were significant differences in $\mathrm{C}$-jun, Caspase-9 and Wnt5a gene expression in the tumor and lung tissues among the NS, PPI-L and PPI-H groups (Fig. 4A-C). For transplant subcutaneous sarcoma, Caspase-9 expression in the drug groups was lower than that in the control group $(\mathrm{P}=0.003$ and $\mathrm{P}=0.010$, respectively), while $\mathrm{C}$-jun expression was higher $(\mathrm{P}=0.043$ and $\mathrm{P}<0.001$, respectively). Wnt5A expression was lower in the high dosage group and exhibited 

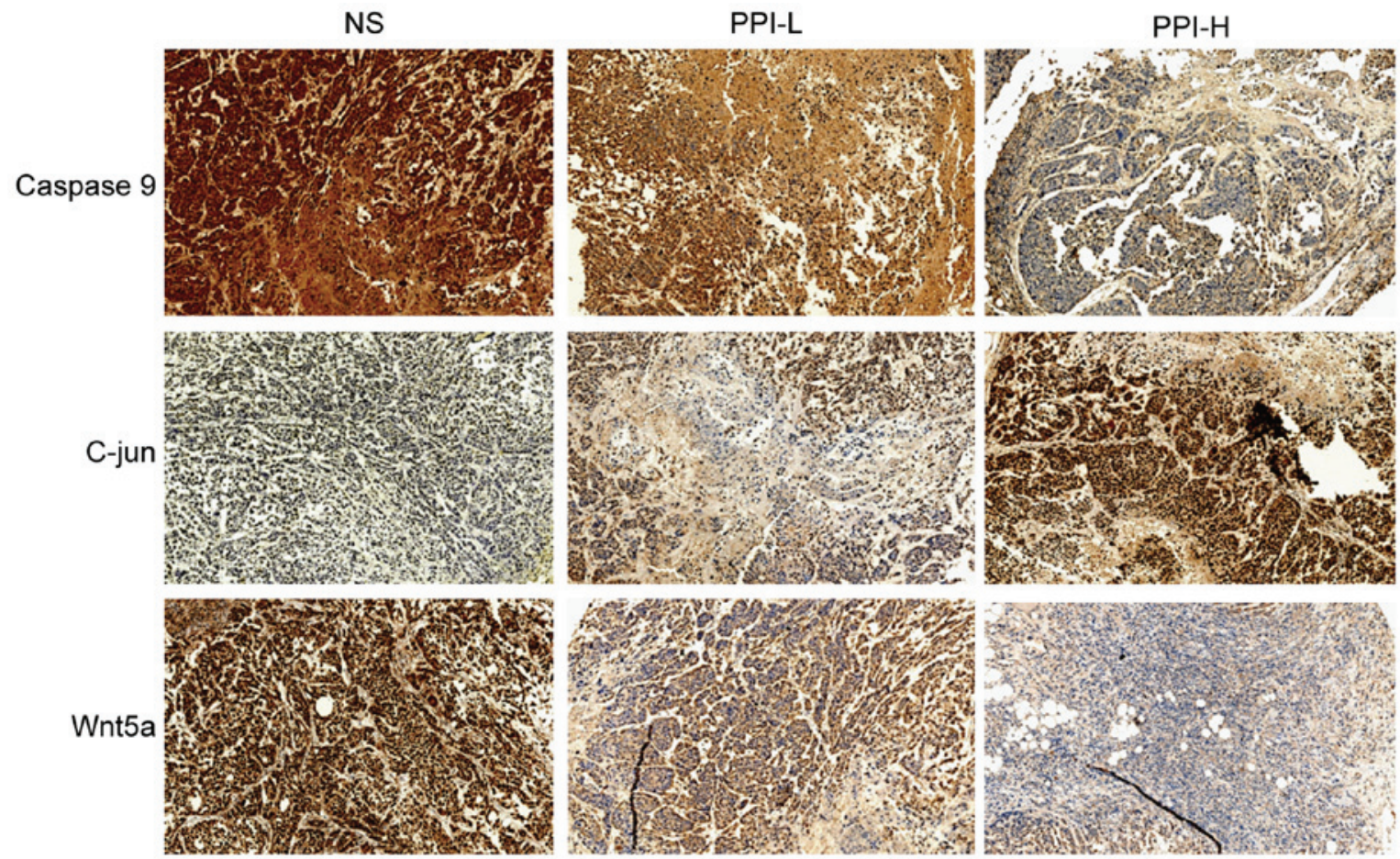

Figure 2. Immunohistochemical staining of Caspase-9, C-Jun and Wnt5a in subcutaneous tumor tissue. Caspase-9 and Wnt5a expression was decreased in the PPI-L and PPI-H groups, while C-jun was increased in the two groups (magnification, x100). PPI, polyphyllin I; NS, normal saline; PPI-H, PPI high concentration; PPI-L, PPI low concentration.
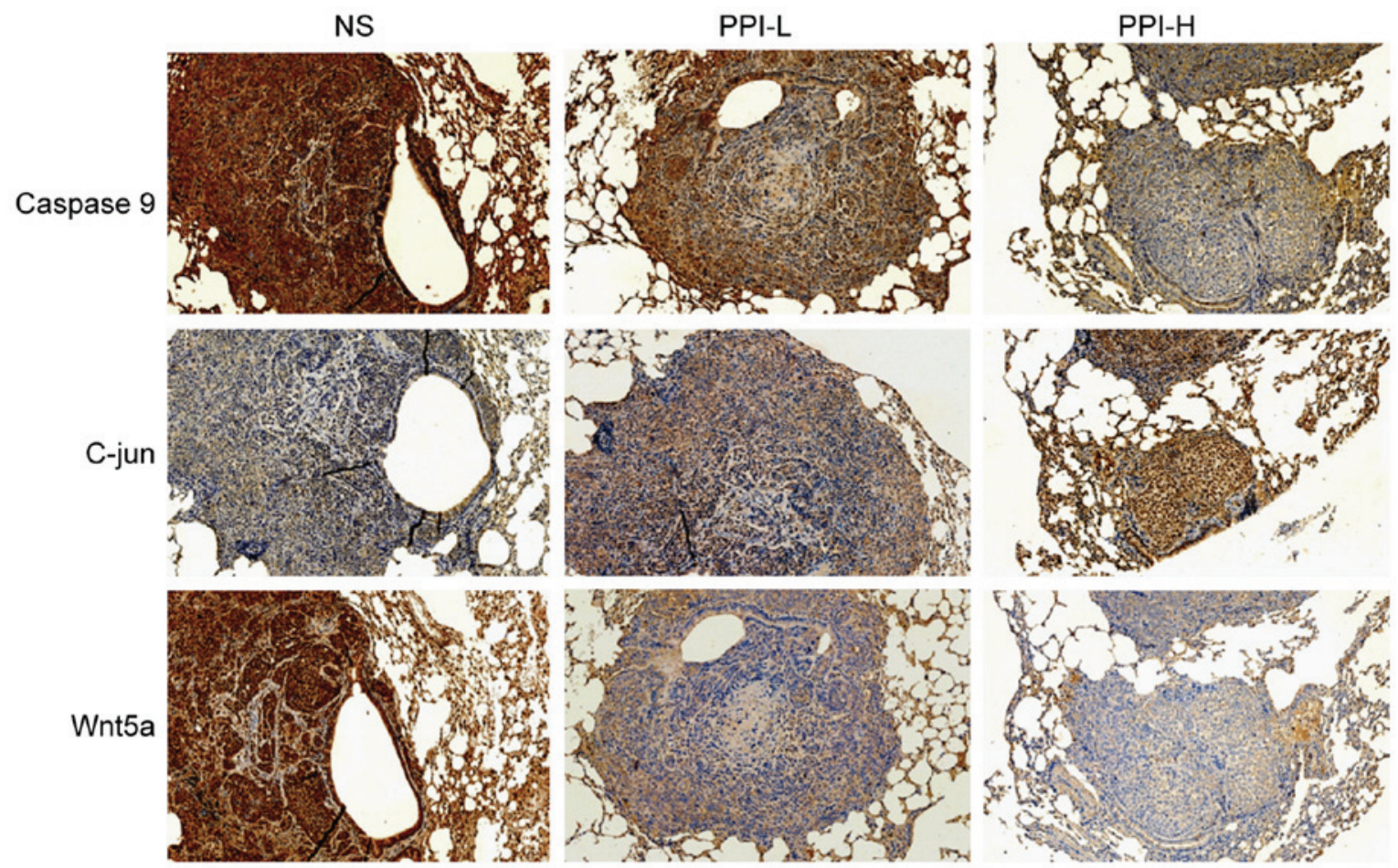

Figure 3. Immunohistochemical staining of Caspase-9, C-Jun and Wnt5a in lung metastatic tumor tissue. Caspase-9 and Wnt5a expression was decreased in the PPI-L and PPI-H groups, while C-jun was increased in the two groups (magnification, x100). PPI, polyphyllin I; NS, normal saline; PPI-H, PPI high concentration; PPI-L, PPI low concentration.

no significant change in the low dosage group $(\mathrm{P}=0.167$ and $\mathrm{P}=0.155$, respectively) (Fig. 4A-C). For the lung tissues, Caspase-9 and C-jun expression in the drug groups was lower than that of the control group $(\mathrm{P}=0.003, \mathrm{P}=0.004$ and $\mathrm{P}=0.005$, respectively), and Wnt5a was lower in the high dosage group, with no significant change in the low dosage group $(\mathrm{P}=0.208)$ (Fig. 4A-C). These results were in compliance with the aforementioned immunohistochemical detection. 
A

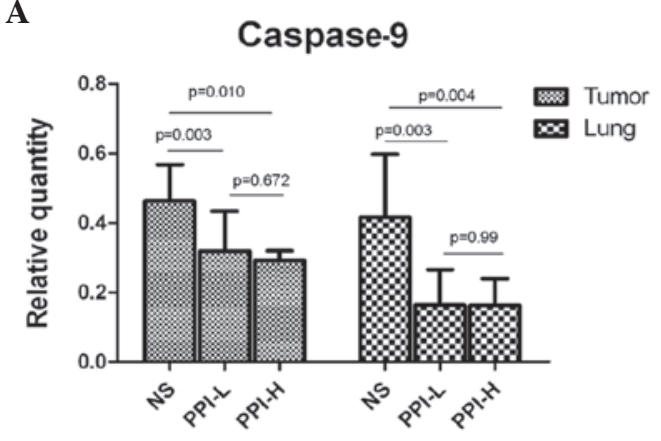

B

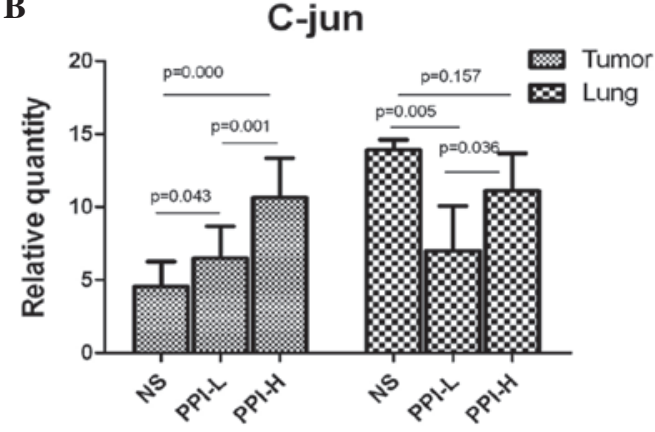

C

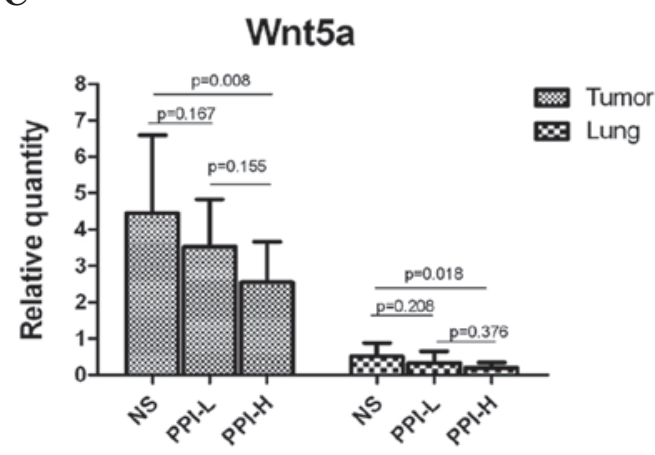

Figure 4. Gene expression of (A) Caspase-9, (B) C-Jun and (C) Wnt5a in subcutaneous and lung metastatic tumor tissues. Gene expression of Caspase-9 and Wnt5a was decreased in the PPI-L and PPI-H groups in the subcutaneous and lung metastatic tumor tissues, while C-jun expression was increased in the subcutaneous tumor tissue but decreased in the lung tumor tissue. P<0.05 was considered to indicate statistical significance. PPI, polyphyllin I; NS, normal saline; PPI-H, PPI high concentration; PPI-L, PPI low concentration.

\section{Discussion}

In the present study, it was found that PPI markedly inhibited the growth of ovarian cancer transplant sarcoma in vivo, which confirmed the antitumor effect on ovarian cancer that was indicated in previous in vitro studies (9). The antitumor effects of PPI in lung cancer have been reported a number of times; for example, PPI increased the radiation sensitivity of gefitinib-resistance lung cancer cell lines (10), PPI markedly inhibited the proliferation and growth of non-small cell lung cancer (11), and PPI suppressed the metastasis of lung cancer (12) in previous studies. More importantly, it was reported that PPI promoted the apoptosis of ovarian cancer cells through the mitochondrial pathway (13). Therefore, these results indicated that PPI exhibits good antitumor effects in vitro and in vivo.

In order to investigate the mechanism of PPI, in our previous in vitro studies, screening of genes by microarray was conducted and it was found that compared with the control group, there were 123 differentially-expressed genes in the cells treated by PPI; 70 were downregulated and 53 were upregulated, and they were associated processes such as apoptosis, proliferation, migration, invasion and angiogenesis. C-jun, Caspase-9 and Wnt5A in particular were validated at the gene and protein levels. The expression of Caspase-9 and Wnt5A decreased with increased dosage of PPI, and the dose-effect association was marked. By contrast, the expression of $\mathrm{C}$-jun increased with increased dosage and prolonged time of drug administration (6). The present study also found that the changes of the three genes in vivo were the same as those in vitro, indicating that these molecules may play important roles in the antitumor effect of polyphyllins.

It was reported that PPI promoted mitochondrial apoptosis, as observed by decreases in the expression of BCL2, increases in the expression of BAX, the release of cytochrome $c$, and the activation and cleavage of Caspase-9 $(10,13)$; while these downstream molecular changes occurred, the upstream changes were not clear. Our studies revealed that the change in expression of C-jun, Caspase-9 and Wnt5A may be associated with the effect of promoting apoptosis. The decreased expression of Caspase- 9 observed in the present study, which is due to cleavage induced by the drug, promoted the apoptosis cascade reaction. There are three main types of Wnt signaling pathways: i) The canonical Wnt/ $\beta$-catenin pathway; ii) the Wnt/JNK pathway (also known as the planar cell polarity pathway); and iii) the $\mathrm{Wnt} / \mathrm{Ca}^{+2}$ pathway, which can be selectively activated by extracellular molecules, thus becoming effector molecules. Activation of JNK can further induce the activity of transcription factors such as C-jun (14,15). C-jun is the specific substrate of JNK, and the activation of this pathway results in the release of apoptosis factor cytochrome $c$ and ultimately mitochondrial apoptosis (16). The present study found that the expression of $\mathrm{C}$-jun increased in the PPI groups, which may be associated with mitochondrial apoptosis. Furthermore, Wnt5A was closely associated with tumor development. The overexpression of Wnt5A may promote the metastasis of tumors and epithelial-mesenchymal transition $(17,18)$. Wnt5A has also been shown to be closely involved in the invasion of prostate cancer cells (19). The present study found significantly decreased expression of Wnt5A when administering PPI, which may suggest its anti-metastatic effect. 
In conclusion, the present study found that PPI exhibited a marked anti-ovarian cancer effect in vivo, with little impact on the body weight of nude mice, which indicated its safety and efficacy, and thus meant that PPI could be considered for further development. The study confirmed expression alterations of three key molecules (Caspase-9, C-jun and Wnt5A) at the molecular level, and that polyphyllins inhibited the expression of Caspase-9 and Wnt5A while promoting the expression of $\mathrm{C}$-jun. The results in this study also provided a basis for further investigating the effects and mechanism of PPI, and will be of great significance.

\section{Acknowledgements}

This study was supported by a grant (no. LQ12H16015) from the Zhejiang Province Natural Science Fund of Youth in China.

\section{References}

1. Siegel R, Miller KD and Jemal A: Cancer statistics, 2015. CA Cancer J Clin 65: 5-29, 2015.

2. Zeng H, Zheng R, Guo Y, Zhang S, Zou X, Wang N, Zhang L, Tang J, Chen J, Wei K, et al: Cancer survival in China, 2003-2005: A population-based study. Int J Cancer 136: 1921-1930, 2015.

3. Sun J, Liu BR, Hu WJ, Yu LX and Qian XP: In vitro anticancer activity of aqueous extracts and ethanol extracts of fifteen traditional Chinese medicines on human digestive tumor cell lines. Phytother Res 21: 1102-1104, 2007.

4. Xiao X, Bai P, Bui Nguyen TM, Xiao J, Liu S, Yang G, Hu L, Chen X, Zhang X, Liu J and Wang H: The antitumoral effect of Paris Saponin I associated with the induction of apoptosis through the mitochondrial pathway. Mol Cancer Ther 8: 1179-1188, 2009.

5. Gu LH, Feng JG, Qian LJ and Ma SL: Research on proliferation inhibitory effect of Paris Saponin I on high metastatic human ovarian cancer cell line HO-8910PM in vitro. Chinese Archives of Traditional Chinese Medicine 30: 2212-2215, 2012 (In Chinese).

6. Gu LH, Feng JG, Xu HY, Luo M and Su D: Polyphyllin I inhibits proliferation and metastasis of ovarian cancer cell line HO-8910PM in vitro. J Tradit Chin Med 33: 325-333, 2013.

7. Shenhua X, Hanzhou M, Lijuan Q, Yongzheng S, Chihong Z, Xiaoshu H, Yongliang G and Shanxing D: Establishment and characterization of a model of highly metastasizing human ovarian cancer transplanted into subcutis of the nude mice. J Exp Clin Cancer Res 14: 387-393, 1995.
8. Chomczynski P and Sacchi N: Single-step method of RNA isolation by acid guanidinium thiocyanate-phenol-chloroform extraction. Anal Biochem 162: 156-159, 1987.

9. Livak and Schmittgen: Analysis of relative gene expression data using real-time quantitative PCR and the $2-\Delta \Delta \mathrm{Ct}$ method. Methods 25: 402-408, 2001.

10. Jiang H, Zhao P, Feng J, Su D and Ma S: Effect of Polyphyllin I on radiosensitivity in a gefitinib-resistant lung adenocarcinoma cell line. Oncol Lett 7: 2059-2064, 2014.

11. Kong M, Fan J, Dong A, Cheng H and Xu R: Effects of polyphyllin I on growth inhibition of human non-small lung cancer cells and in xenograft. Acta Biochim Biophys Sin (Shanghai) 42: 827-833, 2010.

12. Shuli M, Wenyuan G, Yanjun Z, Chaoyi M, Liu Y and Yiwen L: Paridis saponins inhibiting carcinoma growth and metastasis in vitro and in vivo. Arch Pharm Res 34: 43-50, 2011

13. Xiao X, Bai P, Bui Nguyen TM, Xiao J, Liu S, Yang G, Hu L, Chen X, Zhang X, Liu J and Wang H: The antitumoral effect of Paris Saponin I associated with the induction of apoptosis through the mitochondrial pathway. Mol Cancer Ther 8: 1179-1188, 2009.

14. Heasley LE and Winn RA: Analysis of Wnt7a-stimulated JNK activity and cJun phosphorylation in non-small cell lung cancer cells. Methods Mol Biol 468:187-196, 2008.

15. Zhang A, He S, Sun X, Ding L, Bao X and Wang N: Wnt5a promotes migration of humanosteosarcoma cells by triggering aphosphatidylinositol-3 kinase/Akt signals. Cancer Cell Int 14: 15-21, 2014

16. Bates DJ, Lewis LD, Eastman A and Danilov AV: Vincristine activates c-Jun $\mathrm{N}$-terminal kinase in chronic lymphocytic leukemia in vivo. Br J Clin Pharmacol 80: 493-501, 2015.

17. Dissanayake SK, Wade M, Johnson CE, O'Connell MP, Leotlela PD, French AD, Shah KV, Hewitt KJ, Rosenthal DT, Indig FE, et al: The Wnt5A/protein kinase $\mathrm{C}$ pathway mediates motility in melanoma cells via the inhibition of metastasis suppressors and initiation of an epithelial to mesenchymal transition. J Biol Chem 282: 17259-17271, 2007.

18. Taki M, Kamata N, Yokoyama K, Fujimoto R, Tsutsumi S and Nagayama M: Down-regulation of Wnt-4 and up-regulation of Wnt-5a expression by epithelial-mesenchymal transition in human squamous carcinoma cells. Cancer Sci 94: 593-597, 2003.

19. Yamamoto H, Oue N, Sato A, Hasegawa Y, Yamamoto H, Matsubara A, Yasui W and Kikuchi A: Wnt5a signaling is involved in the aggressiveness of prostate cancer and expression of metalloproteinase. Oncogene 29: 2036-2046, 2010. 